\title{
Traffic Signal Control Parameter Calculation Using Probe Data
}

\author{
Toshiya Yoshioka $^{1}$ (D $\cdot$ Hajime Sakakibara ${ }^{2} \cdot$ Robin Tenhagen $^{3} \cdot$ Stefan Lorkowski $^{3} \cdot$ Takashi Oguchi $^{4}$
}

Received: 10 May 2021 / Revised: 24 September 2021 / Accepted: 20 December 2021 / Published online: 31 January 2022

(c) The Author(s) 2022

\begin{abstract}
In signal control, which is one of the main functions of traffic control systems, appropriate signal control parameters are calculated based on the measurement data from vehicle detectors installed on the road. However, the installation and maintenance of vehicle detectors is costly, so realization of a signal control system that can reduce the number of vehicle detectors used while maintaining control level is required. In this paper, we propose a method to calculate signal control parameters without using measurement data from vehicle detectors using traffic information obtained from probe data.
\end{abstract}

Keywords Traffic control system $\cdot$ MODERATO $\cdot$ Load ratio $\cdot$ Probe data

\section{Introduction}

In Japan, the improvement of traffic control systems started in the first half of the 1970's, and has played an important role in society. Its role is to appropriately distribute and guide overcrowded road traffic, and it is composed of two functions, "signal control" to optimize traffic flow and "information provision" to encourage leveling of traffic volume. Traffic control systems recognize traffic conditions

Toshiya Yoshioka

yoshioka-toshiya@sei.co.jp

Hajime Sakakibara

sakakibara-hajime@seiss.co.jp

Robin Tenhagen

Robin.Tenhagen@tomtom.com

Stefan Lorkowski

Stefan.Lorkowski@tomtom.com

Takashi Oguchi

takog@iis.u-tokyo.ac.jp

1 Information Network R\&D Center, Mobility System Department, Sumitomo Electric Industries, Ltd., 1-1-3 Shimaya, Konohana-ku, Osaka 554-0024, Japan

2 System \& Electronics Division, Sumitomo Electric Industries, Ltd., 1-43-5 Sekiguchi, Bunkyo-ku, Tokyo 112-0014, Japan

3 TomTom Traffic B.V., An den Treptowers 1, 12435 Berlin, Germany

4 Advanced Mobility Research Center, Institute of Industrial Sciences, the University of Tokyo, 4-6-1 Komaba, Meguro-ku, Tokyo 153-8505, Japan through vehicle detectors on roads, and implement information provision and signal control according to the situation. Therefore, vehicle detectors are the basis of all traffic management functions. Especially in signal control, which is one of its main functions, the appropriate signal control parameters (cycle length, split, and offset) are calculated based on measurement data from vehicle detectors installed on the approach to every intersection.

Conventional traffic control systems use a control method called "program selection control", which selects the most appropriate signal control parameters for a traffic condition at a given time based on measurement information from vehicle detectors from pre-designed signal control parameters. However, program selection control has problems such as "a lot of work is required to design the parameter" and "it is impossible to cope with large changes in traffic conditions over time". With the purpose of solving these problems, MODERATO (Management by Origin-Destination Related Adaptation for Traffic Optimization) was developed in Japan [1]. This MODERATO control, called "program formation control", automatically generates signal control parameters based on measurement data from vehicle detectors.

As signal control systems have become more sophisticated evolving from conventional program selection control to program formation control, more vehicle detectors have become necessary. In recent years, however, because of the strict budgets of local governments that maintain and operate traffic control systems, not only is it difficult to install a sufficient number of vehicle detectors, but also to maintain and manage the huge number of vehicle detectors that have been 
installed so far. Therefore, realization of a signal control system that can reduce the number of vehicle detectors used while maintaining the necessary control level is required.

On the other hand, in recent years, with the popularization of smartphones, probe data such as the position where vehicles have actually driven and vehicle speed has been collected in large quantities throughout the world. Probe data can be collected even in locations where vehicle detectors are not installed, because the vehicle itself serves as a source of information. Probe data is superior to vehicle detectors in terms of price, wide range of information provision, and is expected to be effective as a means of understanding traffic conditions.

Against this background, this study examined the utilization of probe data as a substitute for vehicle detectors in signal control. In MODERATO control, critical intersections which become bottlenecks of congestion and plural intersections adjoining these critical intersections are controlled in conjunction. Intersection groups controlled in conjunction are called subareas, and intersections in subareas are controlled with common cycle lengths. The cycle lengths applied to each intersection in the subarea and the splits of critical intersections are determined based on an index value called "load ratio" calculated from inflow traffic volume and the number of vehicles in queue estimated by multiple vehicle detectors installed around critical intersections. Therefore, if the load ratio can be calculated from probe data, MODERATO control can be executed even without measurement data from vehicle detectors.

In this paper, we propose a method to calculate load ratio using the average travel time of each approach to an intersection obtained from probe data (hereinafter, probe travel time) and operation information of signal controllers (cycle length and display time of a red signal) without using measurement data from vehicle detectors. In addition, the load ratio calculated from probe data is evaluated for actual routes in Tokyo, and it is confirmed that load ratio can be calculated with the same accuracy as that of vehicle detectors.

\section{Related studies}

In recent years, there have been many studies dealing with probe data, among which, as in this paper, there are some studies considering the utilization of probe data for signal control.

Iwaoka et al. [2] have proposed a method to estimate the delay time caused by signal control from probe travel time, and to design signal control parameters for program selection control using this as an index value.

Blokpoel et al. [3] proposed a way to dynamically change the timing of switching green signals by estimating the queue length by combining the position information of individual probe vehicles with the operation information of signal controllers, and verified its effectiveness by using simulations. However, to achieve the same control level as conventional vehicle detectors, the ratio of probe vehicles to the entire number of vehicles (hereinafter, probe penetration rate) is required to be $30 \%$ or more.

Nagashima et al. [4] proposed a method to estimate the number of vehicles in queue from the position information of individual probe vehicles and the measurement data from vehicle detectors installed near the stop line of the intersection, and to calculate the load ratio by combining this with the inflow traffic volume measured by vehicle detectors. Yoshioka et al. [5] also propose a method to estimate the inflow traffic volume and the number of vehicles in queue from the probe travel time and calculate the load ratio by learning the correlations between the probe travel time and the measurement data from vehicle detectors installed near the stop line with a Neural Network. Both methods assume combined use with vehicle detectors to compensate for low probe penetration.

In this paper, we propose a method to calculate the load ratio without using the measurement data from vehicle detectors by using the probe travel time and the operation information of signal controllers. It is also confirmed that the load ratio can be calculated with the same accuracy as that of the vehicle detector even under a condition where the probe penetration rate is about $10 \%$.

\section{Overview and problems with MODERATO}

Here, we describe the basic approach and problems of MODERATO control, which is widely operated in Japan.

"Flow ratio" is an index value that serves as the basis for parameter calculation. Flow ratio is the inflow traffic volume divided by the saturation flow rate and represents the minimum split required to handle the traffic volume in a non-saturated condition. Flow ratio $(\lambda)$ is obtained by Eq. (1) using the inflow traffic volume (Qin) and the saturation flow rate $(S)$.

$\lambda=\operatorname{Qin} / S$

MODERATO control uses an index value called "load ratio", which is an extension of this flow ratio, in order to include over-saturated conditions as a control target. In order to deal with the over-saturated condition, the amount of inflow traffic volume within the control target time plus the number of vehicles in queue must be considered as the control object, which is called the load traffic volume per unit time. Load ratio $(\rho)$ is the value obtained by dividing this load traffic volume by the saturation flow rate, and is 
obtained by Eq. (2) using the inflow traffic volume (Qin), the number of vehicles in queue $(E)$, and saturation flow rate $(S)$.

$\rho=($ Qin $+E) / S$

As shown in Eq. (2), since load ratio contains the number of vehicles in queue element, a longer green signal can be displayed in the direction in which the traffic jam is occurring. By this, suppressing the lengthening of traffic jams and decreasing the time required to eliminate them can be expected. Incidentally, it is difficult to directly measure the inflow traffic volume ( $Q$ in $)$ when there is a queue. Therefore, as a practical measure, the outflow traffic volume (Qout) is measured at the intersection outflow area, and load ratio is often calculated by Eq. (3) in which Qin in Eq. (2) is substituted by Qout.

$\rho=($ Qout $+E) / S$

Incidentally, in a non-saturated condition, since $E=0$, load ratio is equivalent to flow ratio.

First, load ratio is calculated for each approach, and the load ratio of the approach with the largest load ratio among the approaches processed in the same signal phase is assumed to be the load ratio of that signal phase. That is to say, it is the load ratio of the traffic which requires the longest display time of a green signal among traffic flowing concurrently. In particular, load ratio of the signal phase $i\left(\rho_{i}\right)$ is calculated in Eq. (4) using the load ratio of the approach $j$ to be processed in the signal phase $i\left(\rho_{i j}\right)$.

$\rho_{i}=\max \left(\rho_{i 1}, \cdots \rho_{i j} \cdots, \rho_{i n}\right)$

Here, $n$ represents the number of approaches to be processed at the same time in the signal phase $i$.

Once the load ratio of the signal phase is calculated, load ratio of the intersection $(\hat{\rho})$ is calculated using Eq. (5).

$\hat{\rho}=\sum_{i} \rho_{i}$

In MODERATO control, the intersection load ratio $(\hat{\rho})$ calculated for each critical intersection is substituted into Eq. (6) to calculate the cyclic length $(C)$.

$C=\left(a_{1} \cdot K+a_{2}\right) /\left(1-a_{3} \bullet \hat{\rho}\right)$

Where $K$ represents loss time and $a_{1}, a_{2}$ and $a_{3}$ represent coefficients. Incidentally, Eq. (6) is an extension of the empirical equation of Webster [6] expressed by Eq. (7). Assuming $a_{1}=1.5, a_{2}=5, a_{3}=1$ in Eq. (6), it is consistent with Eq. (7).

$C=(1.5 \cdot K+5) /(1-\hat{\rho})$

In addition, MODERATO control uses a load ratio proportional distribution method in which splits are distributed according to the ratio of each signal phase load ratio to intersection load ratio, and the split of the signal phase $i\left(g_{i}\right)$ is calculated using Eq. (8).

$g_{i}=\rho_{i} / \hat{\rho}$

In over-saturated conditions, this load ratio proportional distribution method treats both main road and minor road congestion equally. That is, MODERATO control can be said to be a system to determine signal control parameters with the aim of equalizing the time required for passing through congestion.

As described above, in MODERATO control, the inflow traffic volume (Qin) (substituted by the outflow traffic volume (Qout) in an over-saturated condition), the number of vehicles in queue $(E)$, and saturation flow rate $(S)$ are estimated from measurement data from vehicle detectors, and load ratio is calculated by substituting these estimates into Eq. (2) (Eq. (3) in the over-saturated condition). Therefore, the problem that the controlled target is limited to an intersection where vehicle detectors are installed exists.

\section{How to calculate load ratio from probe data}

In this section, we describe how to calculate load ratio by the proposed method and the basis for its establishment, dividing the control target intersection into an isolated intersection that is controlled independently of other intersections and a coordinated intersection that is controlled in conjunction with neighboring intersections.

\subsection{When the control target is an isolated intersection}

First, we consider the case where the control target is an isolated intersection. Fig. 1 is a graph representing a travel locus when multiple vehicles pass through the approach from intersection $J_{1}$ to $J_{2}$. The horizontal axis of the graph is the distance from intersection $J_{1}$, and the vertical axis is the travel time. When multiple vehicles pass through this approach, the average delay time per vehicle due to waiting for the traffic light is obtained by dividing the total delay time of all vehicles that pass through the intersection $J_{2}$ after waiting for the traffic light by the number of vehicles. Probe travel time can be considered to include the average delay time per vehicle described above. Therefore, the average delay time per vehicle $(w)$ is calculated using Eq. (9) by subtracting the travel time when this approach is traveled at the regulated speed $(V)$ without waiting for the traffic light from the probe travel time (Tprobe). 


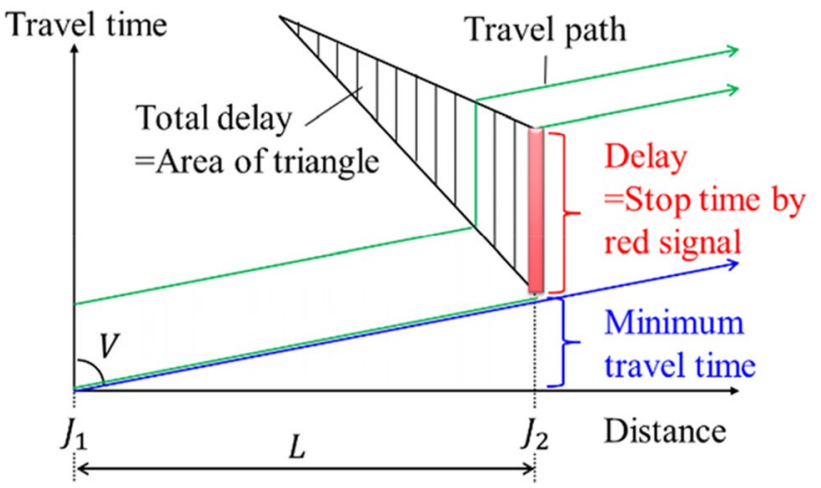

Fig. 1 Travel locus when multiple vehicles pass through approach to isolated intersection

$w=$ Tprobe $-\{L /(V / 3.6)\}$

Here, $L$ is the length of the approach from intersection $J_{1}$ to $J_{2}$.

Figure 2 shows a traffic situation when the approach from intersection $J_{1}$ to $J_{2}$ is non-saturated. In Fig. 2, $S$ is the saturation flow rate, $C$ is the cycle length, $R$ is the display time for the red signal, $Q$ in is the inflow traffic volume, $W$ is the total delay time during one cycle, and $g$ is the time from the start of the green signal until the last vehicle waiting for the traffic light passes through the intersection $J_{2}$. When this approach is non-saturated, the number of vehicles flowing in after the start of the red signal $(=(R+g) \bullet$ Qin $)$ is equal to the number of vehicles flowing out by time $g(=g \bullet S)$. Therefore, $g$ can be calculated using Eq. (10).

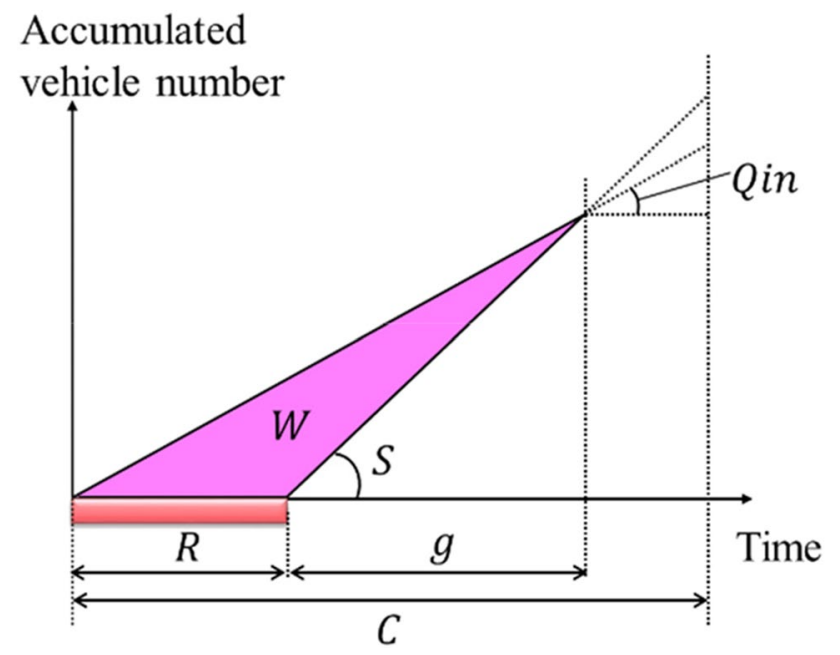

Fig. 2 Traffic situation when target approach is non-saturated

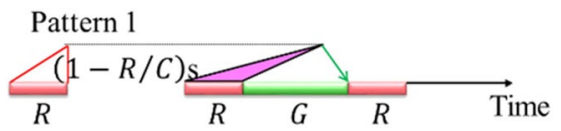

Pattern 2

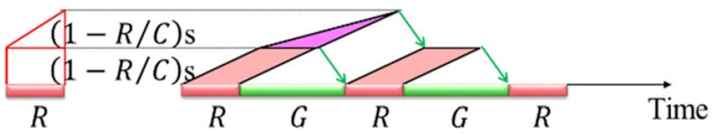

Pattern 3

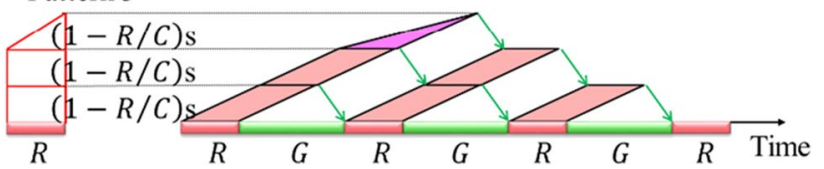

Fig. 3 Traffic situation when target approach is over-saturated

$g=\operatorname{Qin} \bullet R /(S-Q i n)$

Further, $W$ is the area of the triangle in Fig. 2. It is calculated using Eq. (11).

$W=0.5 \cdot\{(R+g) \cdot R \cdot \operatorname{Qin}\}$

Therefore, the average delay time per vehicle $(w)$ is calculated by Eq. (12) by dividing $W$ in Eq. (11) by the number of vehicles $(=C \cdot$ Qin $)$.

$w=0.5 \cdot\{(R+g) \cdot R\} / C$

Here, substituting $g$ in Eq. (10) into Eq. (12) and solving for Qin yields $Q$ in with the coefficients of $S$ as shown in Eq. (13).

$\operatorname{Qin}=\left\{1-R^{2} /(2 \cdot w \cdot C)\right\} \bullet S$

The $w$ in Eq. (13) can be calculated from probe travel time using Eq. (9), but $S$ cannot be measured without vehicle detectors. However, since the formula for calculating the load ratio shown in Eq. (2) has $S$ in the denominator, substituting Qin in Eq. (13) into Eq. (2) cancels out $S$. That is, the load ratio $(\rho)$ when the approach from intersection $J_{1}$ to $J_{2}$ is non-saturated $(w \leq R / 2)$ can be calculated by Eq. (14) using $w$ calculated from probe travel time and the operation information of signal controllers $(R$ and $C$ ).

$\rho=1-R^{2} /(2 \cdot w \cdot C)$

Incidentally, according to Newell et al. [7], the average delay time per vehicle $(w)$ is calculated using the following Eq. (15).

$w=0.5 \cdot(1-(C-R) / C)^{2} \cdot C /(1-Q i n / s)$

When Eq. (15) is rearranged for Qin, the same formula as Eq. (13) is obtained. 
Figure 3 shows a traffic situation when the approach from intersection $J_{1}$ to $J_{2}$ is over-saturated. When all vehicles are in a saturated condition where they can be handled in just one cycle (Pattern 1), the average delay time per vehicle becomes $w=R / 2$ (the mean value of the delay time of the vehicle arriving at the beginning of the display time of the red signal $(=R)$ and the delay time of the vehicle arriving at the end of the display time of the red signal $(=0)$ ), the outflow traffic volume becomes Qout $=(1-R / C) \bullet S$, the number of vehicles in queue becomes $E=0$. On the other hand, when all vehicles are waiting for one cycle (Pattern 2), $w=3 R / 2$ (the mean value of the delay time of the vehicle arriving at the start of the previous display time of the red signal and waiting for one cycle $(=2 R)$ and the delay time of the vehicle arriving at the end of the previous display time of the red signal and waiting for one cycle $(=R))$, Qout $=(1-R / C) \bullet S$ , $E=(1-R / C) \bullet S$. Similarly, when all vehicles are waiting for two cycles (Pattern 3), $w=5 R / 2$, Qout $=(1-R / C) \bullet S$, $E=2 \bullet(1-R / C) \bullet S$. When these relations are expressed by Eqs. (16) and (17), Qout and $E$ are obtained with the coefficients of $S$, respectively.

Qout $=(1-R / C) \cdot S$

$E=\{(w-R / 2) / R\} \cdot(1-R / C) \cdot S$

Again, substituting Qout of Eq. (16) and E of Eq. (17) into Eq. (3), $S$ is canceled. That is, load ratio $(\rho)$ when the approach from intersection $J_{1}$ to $J_{2}$ is over-saturated $(w>R / 2)$ can be calculated by Eq. (18) using $w$ calculated from probe travel time and the operation information of signal controllers $(R, C)$.

$\rho=(1-R / C) \cdot\{1+(w-R / 2) / R\}$

\subsection{When the control target is a coordinated intersection}

Next, we consider the case where the control target is a coordinated intersection. Fig. 4 shows an example where intersection $J_{4}$ is controlled in conjunction with the adjacent intersections $J_{1}, J_{2}$ and $J_{3}$. The average delay time per vehicle $(w)$ when multiple vehicles pass through the approach from intersection $J_{1}$ to $J_{4}$ is difficult to model with a simple triangle, as shown in Fig. 2. Therefore, a traffic simulator is used to simulate the relationship between the inflow traffic volume (Qin) and $w$ in this approach. In the traffic simulator, varying numbers of virtual vehicles are input to the inflow path of the first intersection $\left(J_{1}\right)$ of this approach and $w$ for each generation number is output. The number of virtual vehicles is the number of vehicles normalized by the saturation flow rate $(S)$, for example, increasing as follows: $\operatorname{Qin}=0.1 S \rightarrow$ Qin $=0.2 S \rightarrow$ Qin $=0.3 S \cdots$.

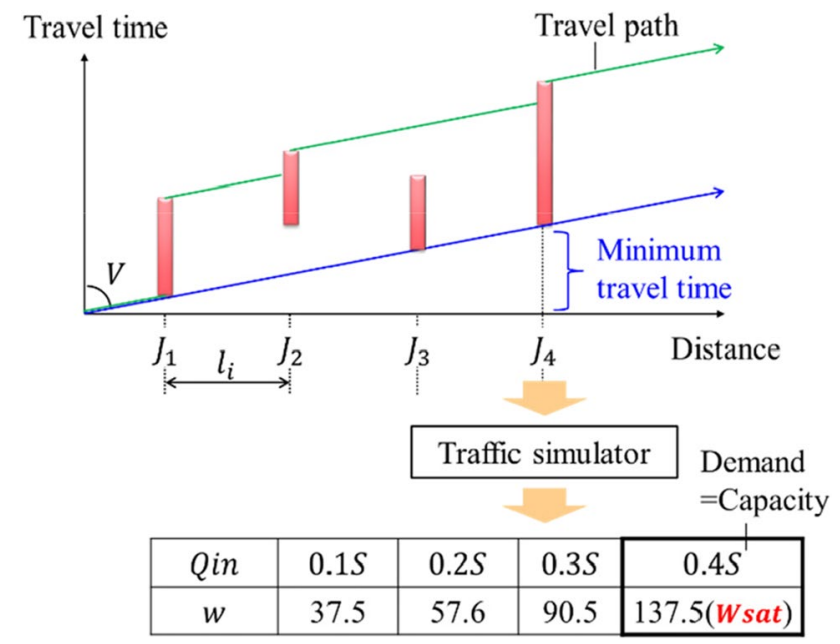

Fig. 4 Correspondence table summarizing relationship between inflow traffic volume and delay time

Then, a correspondence table summarizing the relationship between Qin and $w$ is created, as shown in Fig. 4. For example, when the green signal time rate of the intersection with the smallest green signal time rate on this approach is 0.4, $w=137.5$ corresponding to Qin $=0.4 S$ is the threshold (wsat) for identify traffic conditions (non-saturated or over-saturated). Incidentally, $S$ can not be measured unless by a vehicle detector, but if the values of $Q i n / S$ (flow ratio) are the same, the calculated $w$ will be the same, so any value can be used for $S$.

Next, the average delay time per vehicle $(w)$ is calculated using Eq. (19) by subtracting the travel time when the approach from intersection $J_{1}$ to $J_{4}$ is traveled at the regulated speed $(V)$ without waiting for the traffic lights from the probe travel time of multiple probe vehicles that actually traveled this approach (Tprobe).

$w=$ Tprobe $-\sum_{i}\left\{l_{i} /(V / 3.6)\right\}$

Here, $l_{i}$ is the link length between intersection $i$ and intersection $i+1$.

When the approach from intersection $J_{1}$ to $J_{4}$ is non-saturated ( $w \leq w s a t)$, Qin corresponding to $w$ calculated from probe travel time with the coefficients of $S$ is obtained using the correspondence table shown in Fig. 4. For example, if $w$ was $114 \mathrm{~s}$, the corresponding Qin would be about $0.35 \mathrm{~S}$, between $0.3 S$ and $0.4 S$. Substituting the obtained Qin into Eq. (2), $S$ is canceled and load ratio $(\rho)$ can be calculated. On the other hand, if this approach is over-saturated $(w>w s a t)$, Qin and $E$ are obtained from Eq. (16) and Eq. (20), respectively, with the coefficients of $S$.

$E=\{(w-w s a t) / R\} \cdot(1-R / C) \cdot S$ 
Equation (20) is a formula in which $R / 2$ is replaced by wsat in Eq. (17). Substituting Qin in Eq. (16) and $E$ in Eq. (20) into Eq. (3), $S$ is canceled. Therefore, even when the control target is a coordinated intersection, load ratio $(\rho)$ can be calculated by Eq. (21) using $w$ calculated from probe travel time and the operation information of signal controllers $(R$ and $C)$.

$\rho=(1-R / C) \cdot\{1+(w-w s a t) / R\}$

\section{Evaluation conditions}

To evaluate the effectiveness of the proposed method, we compared load ratio calculated from probe data with load ratio calculated from the measurement data of vehicle detectors on actual routes in Tokyo. This section describes the routes to be evaluated and probe data used.

\subsection{Routes to be evaluated}

Omotesandō Intersection and Hitomikaidō Intersection were selected from the signalized intersections in Tokyo as intersections where sufficient vehicle detectors are installed to provide data for comparison with probe data. Both intersections are coordinated intersections and are controlled by MODERATO. The evaluation was carried out at the most congested approaches of each intersection. Figures 5 and 6 show the overview of each target approach.

As shown in Fig. 5, the approach from intersection "Miyamasuzaka" to "Omotesandō" is an 810-m-long section, with three intersections within the section. On the other hand, as shown in Fig. 6, the approach from intersection "Minamiogikubo" to "Hitomikaidō" is a 1540-m-long section, with four intersections within the section. The regulated speed of both approaches is $60 \mathrm{~km} / \mathrm{h}$.

\subsection{Probe data used for evaluation}

The FCD (Floating Car Data) collected by TomTom were used for this evaluation (https://www.tomtom.com/ja $\mathrm{jp} /$ ) [8]. FCD is collected from PND (Personal Navigation Devices) with the consent of users. FCD consists of GPS measurement position information stored in PND and the time at which it was measured. The collected FCD are processed on TomTom servers and provided in the form of average travel times, aggregated in 1-min units for every section from tens of meters to hundreds of meters in length. As shown in Fig. 7, the average travel time of each approach (Fig. 7-(A)) was generated by summing the average travel time of each section (Fig. 7-(B)) at the current time. In
Fig. 5 Omotesandō approach (North bound)

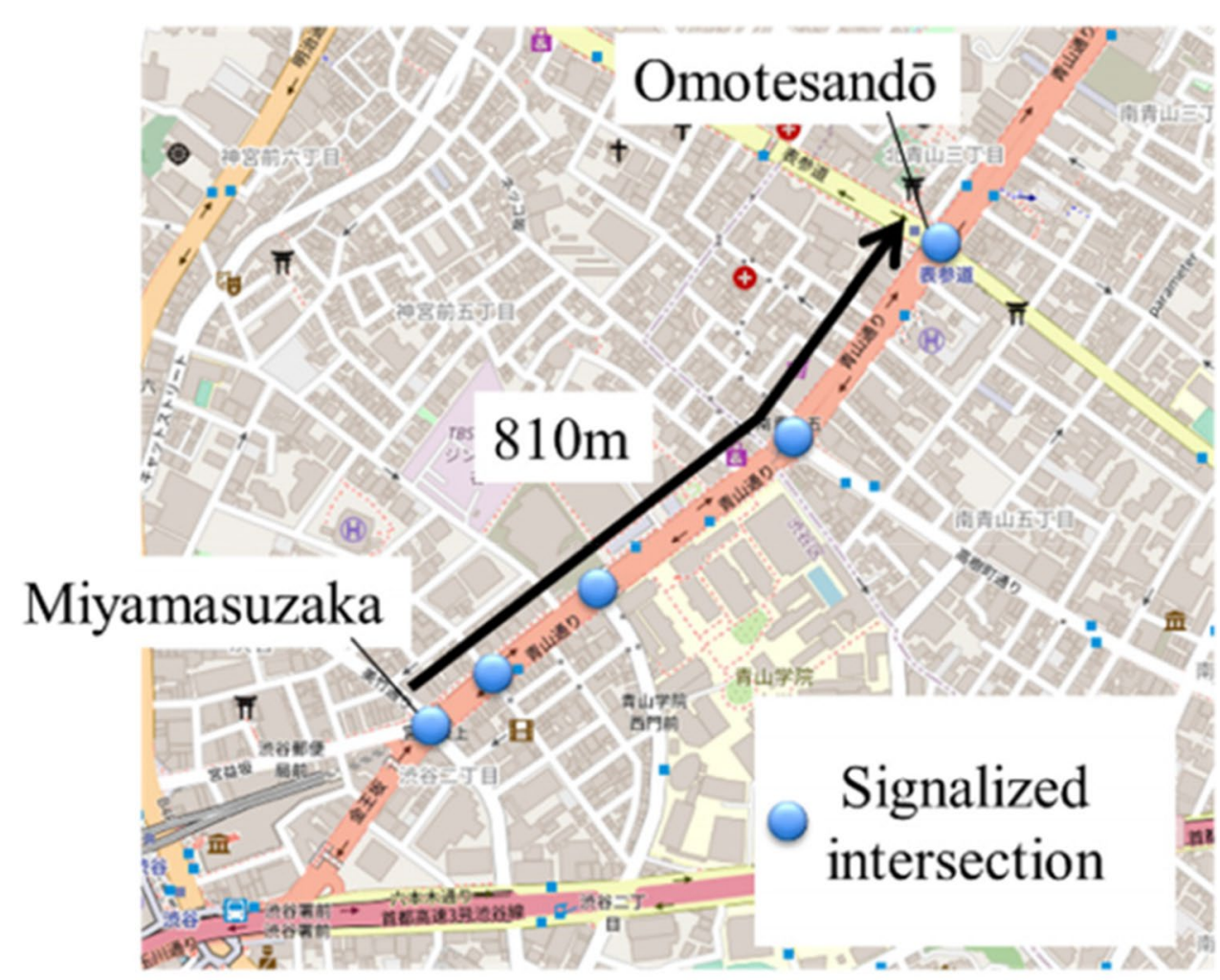

(C) OpenStreetMap contributors 
Fig. 6 Hitomikaidō approach (South bound)

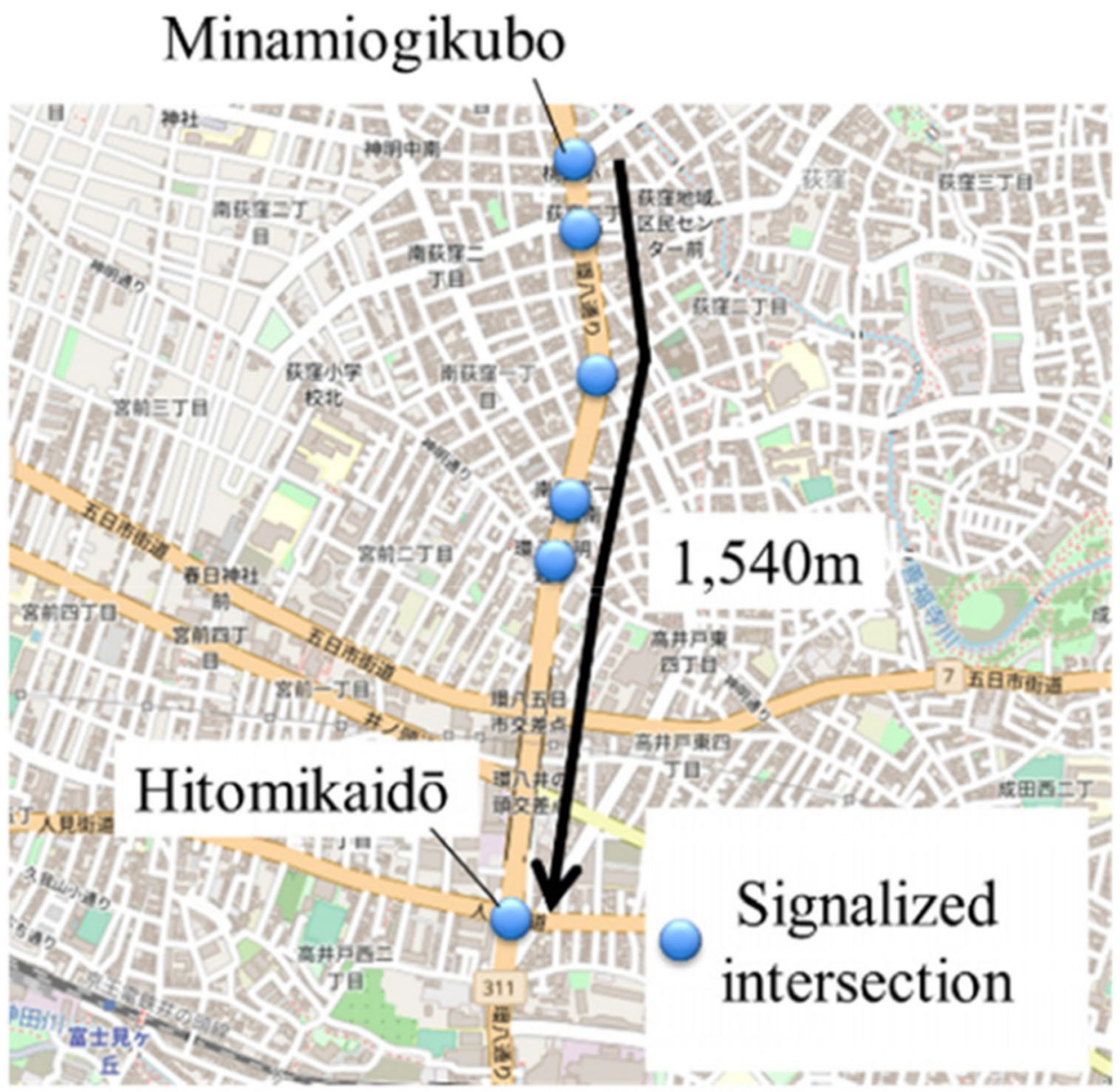

(C) OpenStreetMap contributors

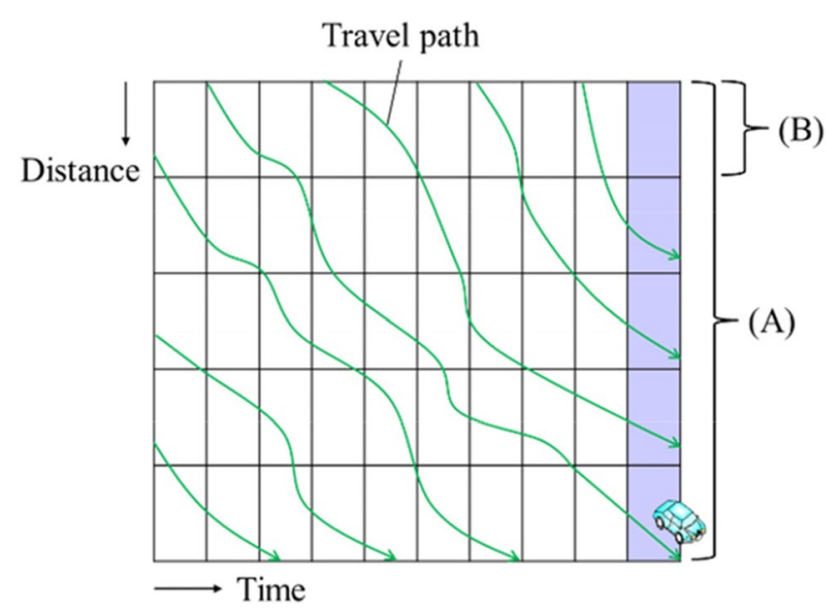

Fig. 7 Relationship between average travel time for each approach (A) and average travel time for each section (B)
Table 1 List of evaluation periods

\begin{tabular}{ll}
\hline Target approach & Evaluation period \\
\hline Omotesandō approach & $2019 / 9 / 10,9 / 16,9 / 18,9 / 20,9 / 27$ \\
Hitomikaidō approach & $2019 / 10 / 24,10 / 25,10 / 28,10 / 29,10 / 30$ \\
\hline
\end{tabular}

addition, the evaluation period was set at five weekdays for each approach as shown in Table 1.

The understanding of traffic conditions is considered to be more accurate as the probe penetration rate rises. The probe penetration rate was estimated from the traffic volume measured by vehicle detectors and the number of probe data collected by TomTom in each target approach. The results indicate the probe penetration rate during daytime was about $12 \%$. This is a value equivalent to the uplink rate of infrared beacons in Tokyo, which are currently collected by the traffic control system of the Metropolitan Police Department and are actually utilized for signal control and information provision $[9,10]$. The display time of the green signal during the daytime on the Omotesandō approach was about $40 \mathrm{~s}$. 
Assuming that the saturation flow rate of this approach is 0.5 vehicles/s, the outflow traffic volume per cycle in a saturated condition is 20 vehicles. In other words, a probe penetration rate of $12 \%$ means that the number of probe vehicles per cycle is about 2 vehicles, so it can be said that a minimum amount can be ensured in measuring probe travel time. Conversely, if the probe penetration rate falls below $12 \%$, probe travel times cannot be measured correctly, making it difficult to calculate load ratio.

\section{Evaluation results}

\subsection{Determination of traffic conditions}

In order to calculate the load ratio, it is important to determine correctly whether the traffic condition is non-saturated or over-saturated. In the proposed method, the traffic condition is determined using the average delay time per vehicle $(w)$ calculated from probe travel time and the threshold (wsat) calculated using the traffic simulator. Figure. 8 shows the determination results at the Omotesandō approach. In the upper half of Fig. 8, the horizontal axis represents the time, and the vertical axis represents the queue length estimated by the method in previous research by Ito et al. [11] using measurement data from vehicle detectors. On the other hand, in the lower half of Fig. 8, the horizontal axis represents the time, the vertical axis represents the average delay time calculated from probe travel time, and the red line represents
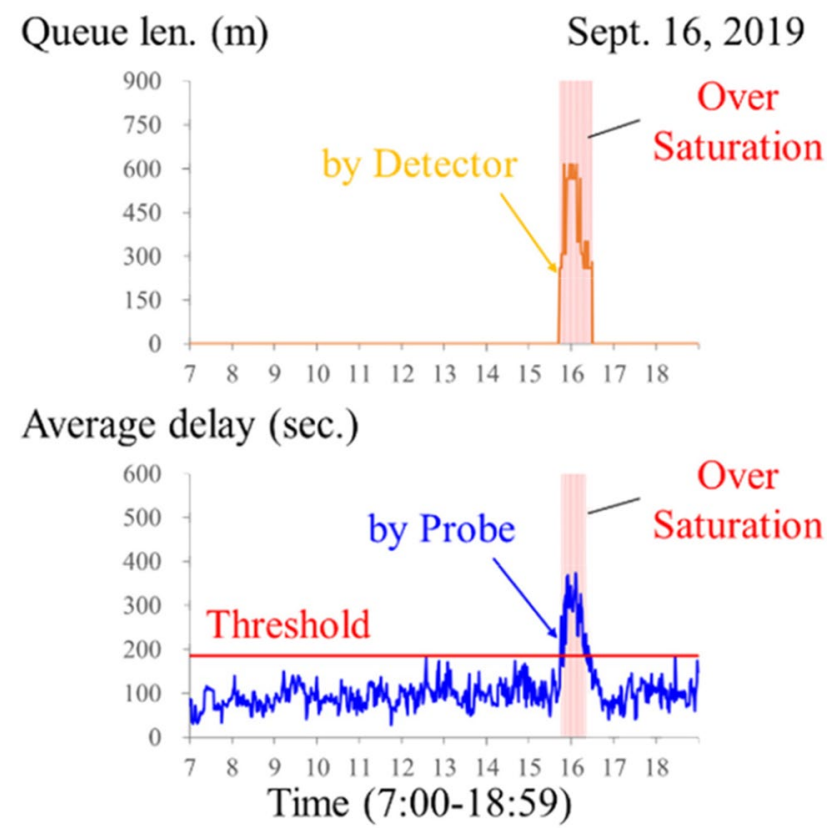

Fig. 8 Determination results of traffic condition at Omotesandō approach the threshold. The period when the average delay time is above the threshold value is determined to be over-saturated, the period when this is not the case is determined to be nonsaturated. As shown in Fig. 8, the period at which oversaturation was determined using probe data coincided with the period at which the queue actually occurred. Further, Fig. 9 shows the determination results at the Hitomikaido approach. Similar to the Omotesandō approach, the period at which over-saturation was determined using probe data coincided with the period at which the queue actually occurred. From these results, it can be said that even at intersections where vehicle detectors are not installed, if probe data is used, traffic conditions (non-saturated or over-saturated) can be determined correctly.

\subsection{Load ratio in a non-saturated condition}

When the traffic condition of the target approach is nonsaturated, inflow traffic volume is estimated from the average delay time calculated from probe data using the correspondence table of the inflow traffic volume (Qin) normalized by the saturation flow rate $(S)$ and the average delay time $(w)$, as shown in Fig. 4. At this time, we expected that when the traffic volume input to the traffic simulator was changed, the output delay time would change as well. However, as shown in Fig. 10-(A), the output value of the delay time hardly changed at the Omotesandō approach. This is considered to be due to the impact of an offset pattern. At the Omotesandō approach, the offset is designed so that the first vehicle of a

Queue len. (m)

Oct. 30,2019

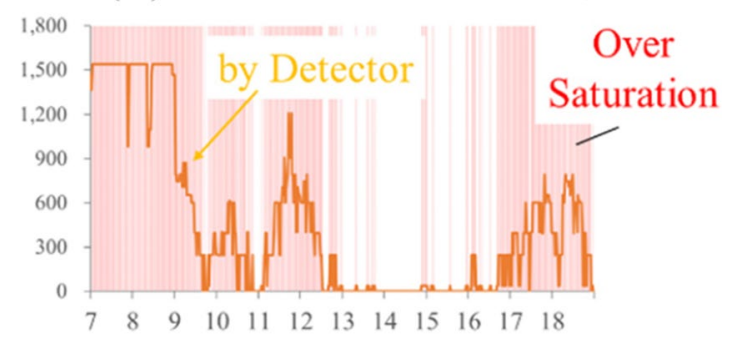

Average delay (sec.)

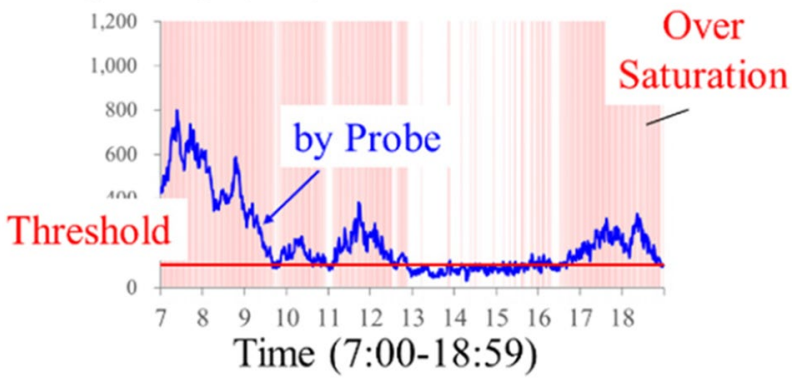

Fig. 9 Determination results of traffic condition at Hitomikaidō approach 
(A) Present offset

\begin{tabular}{|c|c|c|c|}
\hline Qin & $0.21 S$ & $0.24 S$ & 0.27 S \\
\hline$w$ & 180.7 & 182.9 & $184.8($ W sat $)$ \\
\hline
\end{tabular}

(B) Reviewed offset

\begin{tabular}{|c|c|c|c|}
\hline Qin & $0.21 S$ & $0.24 S$ & $0.27 S$ \\
\hline$w$ & 69.1 & 81.7 & 101.2 \\
\hline
\end{tabular}

Fig. 10 Correspondence table between traffic volume and average delay time at Omotesandō approach for each offset pattern

car group that passes through the Miyamasuzaka intersection arrives during the first half of the display time of the red signal of the Omotesando intersection. That is, even if the traffic volume changed, the average delay time per vehicle hardly changed because most vehicles were stopped by the red signal. On the other hand, Fig. 10-(B) is a correspondence table of inflow traffic volume and average delay time when the offset is set so that the second half of the vehicle group arrives in the first half of the display time of a red signal. It can be seen that the sensitivity of the average delay time to the change in traffic volume is higher than that in the case of the present offset pattern. That is, in the case of coordinated intersections, the sensitivity of the average delay time to changes in traffic volume may be higher or lower depending on the design of the offset pattern. At intersections with low sensitivity, it may be difficult to calculate the load ratio in a non-saturated condition using only probe data.

\subsection{Load ratio in an over-saturated condition}

When the traffic condition of the target approach is oversaturated, the load ratio is calculated using the above Eq. (21). Figure 11 shows a comparison of the load ratio calculated from probe data and the load ratio calculated from the measurement data from vehicle detectors in the Omotesandō approach. The horizontal axis of the graph represents the time, and the vertical axis represents the load ratio. Incidentally, in this section, the average load ratio per day was calculated by combining the load ratio calculated for each day of the evaluation period. The green range in the correlation diagram represents the load ratio in just the saturated condition and is the allowable range set in consideration of the average installation interval of vehicle detectors. If the data were within this range, it was determined that the accuracy of both data were in agreement. As shown in Fig. 11, all the data are within the allowable range, and the load ratio calculated from probe data matches the load ratio calculated from the measurement data from vehicle detectors. On the other hand, Fig. 12 shows the comparison results at the Hitomikaido approach. $96 \%$ of the total data fall within the allowable range, and the tendencies of both data are generally consistent. The $4 \%$ of data that is out-of-tolerance is the data for morning peak times, where much of it has a large queue length. As shown in Fig. 13, in MODERATO control, the farther away from the stop line, the wider the installation spacing of the vehicle detectors, and with this, the estimation accuracy of the queue tail also decreases. Therefore, when the queue becomes longer than a certain length, it is impossible to capture the minute fluctuations of traffic demand, and change in the calculated load ratio slows. On the other hand, the delay time calculated from probe data varies depending on the degree of the queue. Therefore, the difference between the two data is considered to be larger during the morning peak time. From these results, it can be said that even at intersections where vehicle detectors are not installed, if probe data is used, the load ratio in an over-saturated condition can be calculated with an accuracy equal to or higher than that of the conventional system.
Fig. 11 Comparative results of load ratio when Omotesandō approach is over-saturated
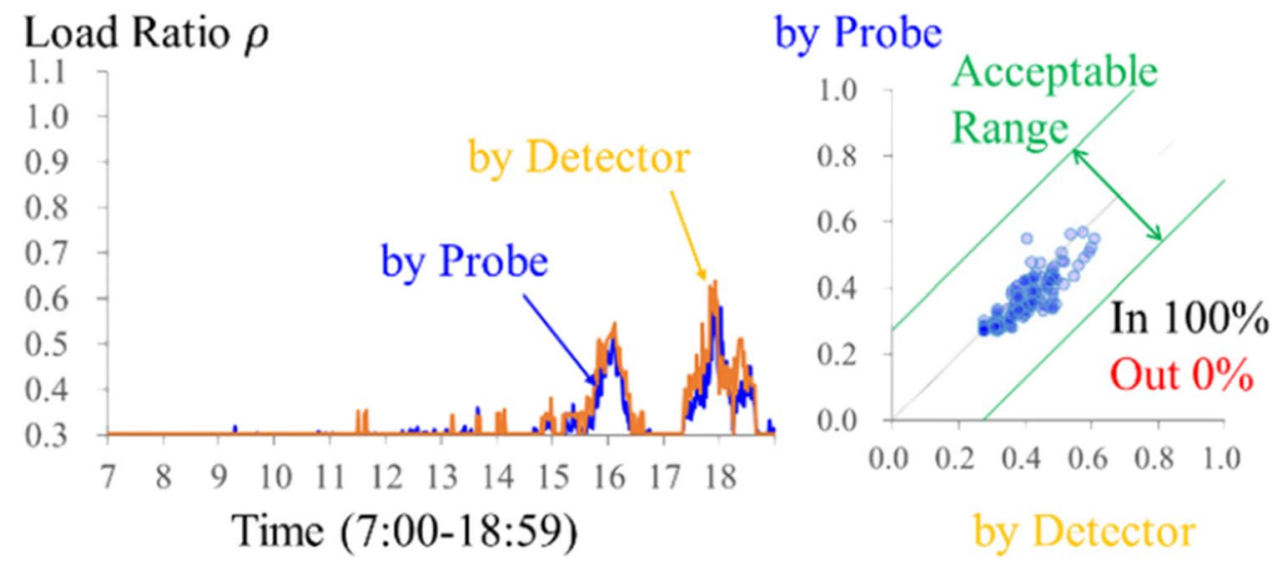
Fig. 12 Comparative results of load ratio when Hitomikaidō approach is over-saturated

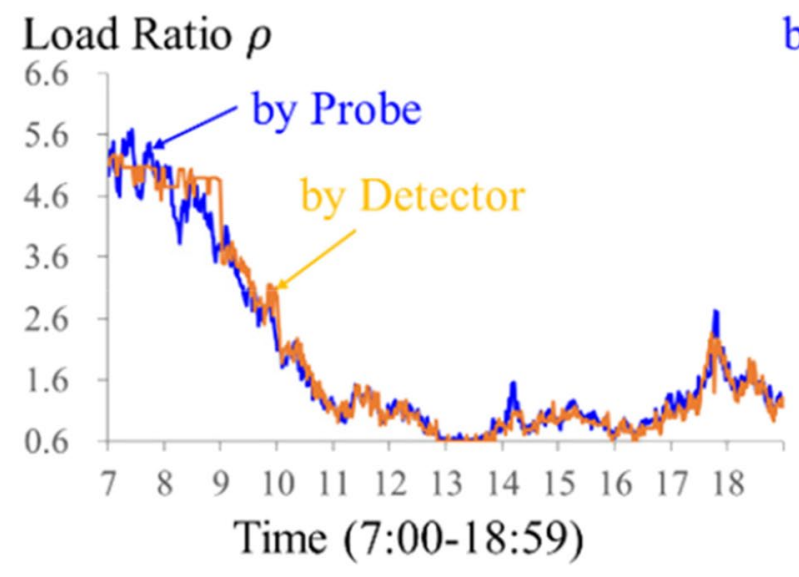

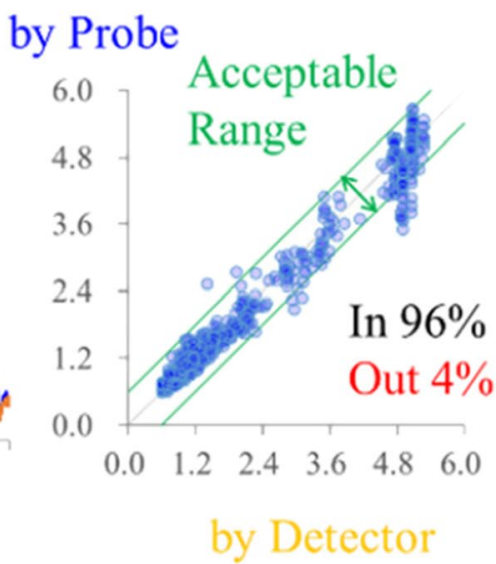

\section{Conclusion}

In MODERATO control which is widely operated in Japan, the traffic flow is optimized by coordinated control of critical intersections which become a bottleneck for traffic jams and multiple intersections adjacent to those intersections. MODERATO control determines signal control parameters based on a traffic index called load ratio, which is calculated using the inflow traffic volume and the number of vehicles in queue measured by vehicle detectors installed on roads. Therefore, there was a commonly held preconception that vehicle detectors would be required to perform MODERATO control. In this paper, contrary to this preconception, we propose a way to execute MODERATO control without using the measurement data from vehicle detectors by using probe data. Specifically, it is a method for calculating load ratio from the average travel time obtained from probe data and the operation information of signal controllers. As a result of the evaluation of the proposed method at intersections in Tokyo, a problem was found when the target intersection is a coordinated intersection and the traffic condition is non-saturated. The sensitivity of average travel time to changes in traffic volume may be low because of an offset pattern, making it difficult to calculate the load ratio from

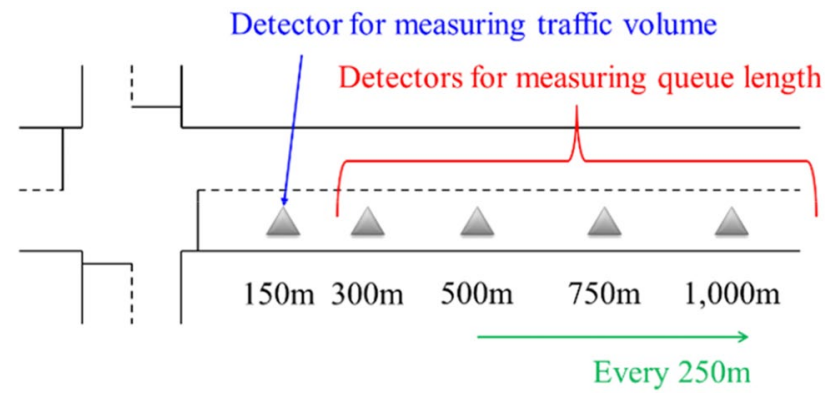

probe data only. On the other hand, it was confirmed that when the traffic condition is over-saturated, load ratio could be calculated using probe data with an accuracy equal to or higher than that of the conventional system. This means that even at intersections where vehicle detectors are not installed or removal of vehicle detectors is being considered, if probe data is used, MODEARATO control can be executed to allocate longer display time of the green signal in the direction in which the queue is occurring, thus suppressing the lengthening of traffic jams and a decrease in the time required to eliminate them can be expected. The proposed method can be implemented by simply replacing input data to the traffic control system from conventional measurement data from vehicle detectors with probe data. There is no need to significantly change the mechanism and equipment of current signal control. In the future, the signal control system will be improved according to the characteristics of probe data, but the proposed method is useful at a time when the means of measuring traffic flow is shifting from conventional vehicle detectors to probe data. In the future, the verification will be carried out in more places, and problems for practical application will be solved. The next goal is therefore implementation in traffic control systems.

Open Access This article is licensed under a Creative Commons Attribution 4.0 International License, which permits use, sharing, adaptation, distribution and reproduction in any medium or format, as long as you give appropriate credit to the original author(s) and the source, provide a link to the Creative Commons licence, and indicate if changes were made. The images or other third party material in this article are included in the article's Creative Commons licence, unless indicated otherwise in a credit line to the material. If material is not included in the article's Creative Commons licence and your intended use is not permitted by statutory regulation or exceeds the permitted use, you will need to obtain permission directly from the copyright holder. To view a copy of this licence, visit http://creativecommons.org/licenses/by/4.0/.

Fig. 13 Example of vehicle detector placement in MODERATO 


\section{References}

1. Sakakibara, H., Usami, T., Itakura, S., and Tajima, T., "MODERATO (Management by Origin-DEstination Related Adaptation for Traffic Optimization)", The $6^{\text {th }}$ World Congress on ITS (Toronto, 1999)

2. Iwaoka, K., Asada, S., Niikura, S., and Kamata, K., "Field Experiment of Offline Traffic Signal Control Using Probe Data Collected via Infrared Beacons", The $20^{\text {th }}$ World Congress on ITS (Tokyo, 2013)

3. Blokpoel, R., Vreeswijk, J., "Use of probe vehicle data in traffic light control", The $6^{\text {th }}$ Transport Research Arena (Warsaw, 2016)

4. Nagashima, Y., Hattori, O., and Kobayashi, M., "Improvement of Traffic Signal Control Using Probe Data”, SEI Technical Review, No. 78, 2014

5. Yoshioka, T., Matsumoto, H., Nagashima, Y., and Yamamoto, T., "Traffic Signal Control with Fewer Detectors Using Probe Data", The $26^{\text {th }}$ World Congress on ITS (Singapore, 2019)

6. Webster, F.V., "Traffic Signal Settings", Road Research Technical Paper, No. 39, 1958

7. Newell, G. F., "Theory of Highway Traffic Signals", Research Report, UCB-ITS-RR-89-7, 1989

8. Meijer, A., Arem, B. V., Salomons, M., Krootjes, P., and Cohn, N., "Probe Data from Consumer GPS Navigation Devices for the Analysis of Controlled Intersections", ITS America 22nd Annual Meeting \& Exposition (Washington D. C., 2012)

9. Hashimoto, S., Senga, M., Hayami, M., and Yamazaki, K., "Effects of and Future Plans for MPD Traffic Control System / Traffic Data Collection and Analysis Functions", The $20^{\text {th }}$ World Congress on ITS (Tokyo, 2013)

10. Sakakibara, H., Ogami, H., Noda, M., and Kawasugi, K., "Reproducibility Enhancements of Traffic Simulator and ITS Performance Evaluation", The $21^{\text {st }}$ World Congress on ITS (Detroit, 2014)

11. Ito, T., and Tani, K., "Effective Verification of Traffic Information to Maintain the Accuracy of Information Provision", The $12^{\text {th }}$ World Congress on ITS (San Francisco, 2005)

Publisher's Note Springer Nature remains neutral with regard to jurisdictional claims in published maps and institutional affiliations.

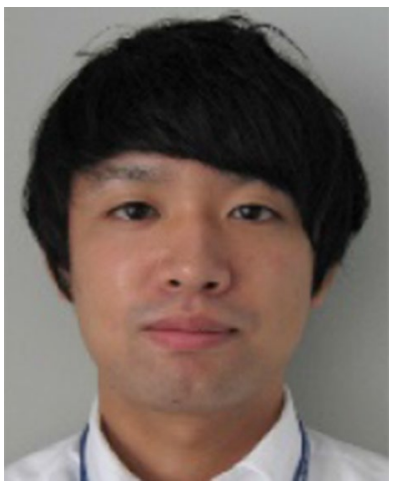

Toshiya Yoshioka entered Sumitomo Electric Industries in 2014 and have worked on the development of signal control methods and techniques in traffic control systems ever since.

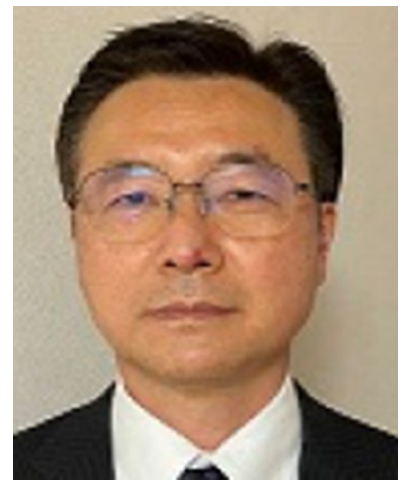

Hajime Sakakibara entered Sumitomo Electric Industries in 1988 and have worked on the development of signal control methods and techniques in traffic control systems ever since.

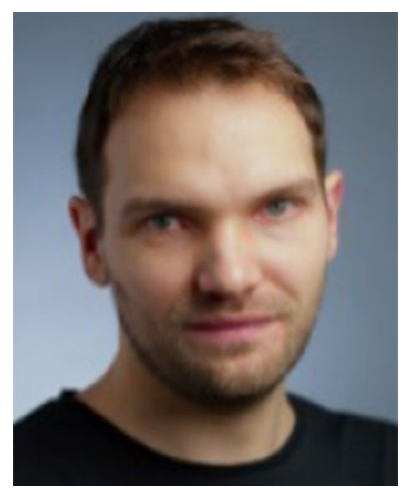

Robin Tenhagen entered TomTom Traffic B.V. in 2015 and has worked in live traffic engineering ever since.

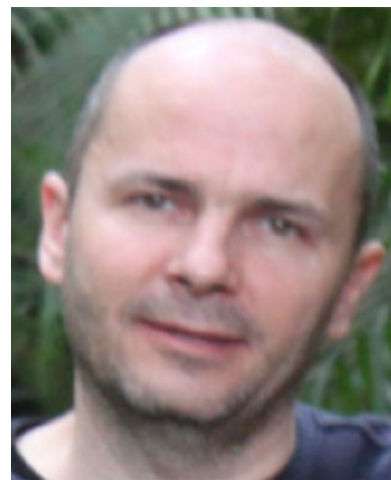

Stefan Lorkowski entered TomTom Traffic B.V. in 2006 and has worked on the engineering of TomTom's live Traffic service ever since.

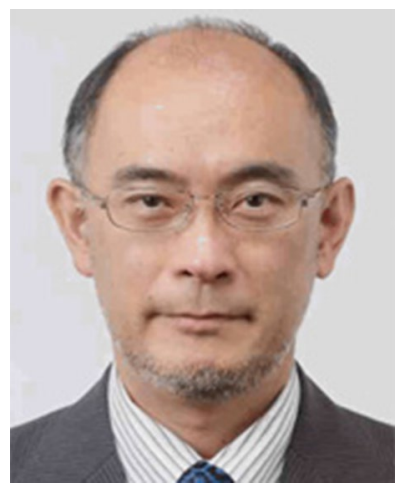

Takashi Oguchi got Ph.D. from the Univ. of Tokyo in 1993, and joined current position in 2011 after working in Nissan Motor and Tokyo Metropolitan Univ. His specialty is Traffic Management and Control. 